\title{
Guest Editors' Introduction to the Thematic Issue on Family Economic Issues in Developing and Transition Economies
}

\author{
Zhuo Chen · Jack W. Hou
}

Published online: 3 March 2009

(C) U.S. Government 2009

This special issue of the Journal of Family and Economic Issues is a joint effort between the editorial office of the Journal and the Board of Directors (2007-2008) of the Chinese Economists Society. It has seven articles that address topics related to family economic issues in developing and transition economies. Initial submissions were solicited and selected from 161 papers originally presented at the 2008 Annual Conference of the Chinese Economists Society in Tianjin, China, on April 17-19, 2008. Six papers in this issue fall in this category. In addition, authors of one paper were not able to attend the Tianjin conference to present their paper but were invited to submit their paper because of the relevance of the paper's topic to the theme of this special issue. All submissions went through the standard double-blind peer-review process.

The papers cover topics include health, consumption, gender segregation in occupation, and immigrant remittance in seven developing and transition economies.

Assessing effectiveness of public programs is the quintessence of program evaluation research. Using data from the Matlab Health and Socio-economic Survey 1996, Chaudhuri looks into the spillover effects of a reproductive

Disclaimer The findings and conclusions in this report are those of the authors and do not necessarily represent the views of the Centers for Disease Control and Prevention (CDC).

\section{Z. Chen $(\square)$}

Office of Workforce and Career Development,

Centers for Disease Control and Prevention,

1600 Clifton Rd NE, Atlanta 30333, GA, USA

e-mail: zchen1@cdc.gov

J. W. Hou

Department of Economics, California State University,

Long Beach 90840-4607, CA, USA

e-mail: jackhou@csulb.edu health program on the bodyweight of elderly who were not targeted by the program but were in households with at least a member participating in the program. Examining the externalities through spillover effects within a household is certainly a worthy contribution to the literature.

The paper by Cho and colleagues sheds light on the interaction between academic achievement and body weight by using an econometric model that accommodates reverse causality and the sampling design. Note that, although higher body weight in Matlab, Bangladesh indicates better nutrition, the binary indicator of overweight used by Cho and colleagues suggests over-nutrition and it is negatively associated with academic achievement in Korea.

With emerging and integrating commodity markets, developing and transition economies present unique opportunities and challenges for researchers examining consumer economics. The paper by Pan, Fang, and Rejesus evaluates the effect of price uncertainties on farm income, and consequently, calories intake among a sample of rural Nepalese households. They find a deleterious effect of the price uncertainties, which underlines the importance of well-functioning commodity markets in developing economies.

Examining factors that affect alcohol-purchase decisions and expenditures by ethnicity in Malaysia is the topic in the paper by Tan, Yen, and Nayga. Their findings indicate that the associations between socioeconomic factors and alcohol consumption vary across ethnicity groups and have critical policy relevance.

Mokhtari and Asgary use data from the Soviet Interview Project to examine the effect of consumer goods shortage on fertility. Their analyses have further improved our understanding of how family decisions can be affected by external forces, including public policies and economic conditions. 
Fuess and Hou investigate the impact of rapid economic development on gender segregation by occupation and job status in Taiwan. They outline the trend of such segregation and offer thoughtful explanations of why the rising segregation index might be part of the trajectory of economic evolution. Further research with micro-level data would identify socioeconomic determinants of gender segregation by occupation. Similar studies that use data from other economies, including mainland China, might provide critical policy insights.

Gupta and Hegde present an exploratory study of the relationship between household characteristics and immigrant remittances by using a small-sample survey. They argue that policy instruments might be used to induce increased immigrant remittance to the home country. Although they cited Indian Government's action of reducing barriers for overseas Indians to acquire property within India and facilitating financial transactions through Internet banking, the fact that India has allowed dual citizenship recently might well be another reason that India is leading all other countries in receiving immigrant remittance. Further research on this perspective might be of interest, given availability of data before and after the policy change.

Before you embark on this intellectual trek in the seven developing and transition economies, we would like to thank the reviewers who have put in so much work and provided many detailed comments and valuable suggestions. Undoubtedly, the papers in this special issue, as well as the research community, have benefited from their dedicated service to the profession. The reviewers are as follows:

Asfaw, Abay, G. at the U.S. Centers for Disease Control and Prevention,

Chern, Wen S. (Charlie) at National Chung Cheng University, Taiwan,

Chou, Shin-yi at Lehigh University, Bethlehem, Pennsylvania,

Fang, Xiangming at the U.S. Centers for Disease Control and Prevention,
Jacobs, Krista at the International Center for Research on Women, Washington D.C.,

Kim, In Kyu at the Milken Institute, Santa Monica, California,

Ouyang, Lijing at the U.S. Centers for Disease Control and Prevention,

Pan, Suwen at Texas Tech University, Lubbock, Texas,

Prabhu, Vimalanand S. at the U.S. Centers for Disease Control and Prevention,

Qiao, Xue (Sherry) at Tsinghua University, Beijing, China, Sengupta, Sanchita at Iowa State University, Ames, Iowa, Shemyakina, Olga at Georgia Institute of Technology, Atlanta, Georgia,

Shrestha, Ram at the U.S. Centers for Disease Control and Prevention,

Shrestha, Sundar at the U.S. Centers for Disease Control and Prevention,

Sotnikov, Sergey at the U.S. Centers for Disease Control and Prevention,

Tseng, Samuel H. at Resolution Economics, Inc., Beverly Hills, California,

Wang, Siyan at University of Delaware, Newark, Delaware,

Wu, Yu (Yuna) at Amgen Inc., Thousand Oaks, California.

Zhuo Chen would also like to thank colleagues at the Office of Workforce and Career Development, the Centers for Disease Control and Prevention, for continuing support and encouragement. Both guest editors would like to express their appreciation for support to the remainder of the members of the Board of Directors (2007-2008) and the general membership of the Chinese Economists Society, and for hospitality to Nankai University, which cohosted the annual conference. Lastly, we thank the editor of this journal, Professor Jing Jian Xiao, for the opportunity to work on the special issue. Zhuo Chen particularly wants to thank Professor Xiao for his guidance and comments whenever requested. 